AFRL-RD-PS-

AFRL-RD-PS-

TR-2014-0036

\title{
ADVANCED CONTROL FILTERING AND PREDICTION FOR PHASED ARRAYS IN DIRECTED ENERGY SYSTEMS
}

\author{
James Steve Gibson
}

University of California, Los Angeles

Office of Research Administration

11000 Kinross Avenue, Ste 102

Los Angeles, CA 90095-2000

31 July 2014

Final Report

APPROVED FOR PUBLIC RELEASE; DISTRIBUTION IS UNLIMITED.

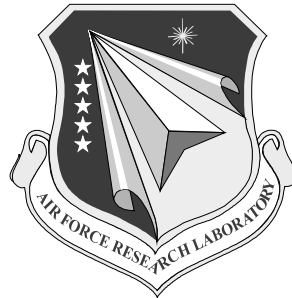

AIR FORCE RESEARCH LABORATORY

Directed Energy Directorate

3550 Aberdeen Ave SE

AIR FORCE MATERIEL COMMAND

KIRTLAND AIR FORCE BASE, NM 87117-5776 
This page intentionally left blank.

Approved for public release; distribution unlimited. 


\section{Notice Page for Approved for Public Release TR (Unlimited Distribution) NOTICE AND SIGNATURE PAGE}

Using Government drawings, specifications, or other data included in this document for any purpose other than Government procurement does not in any way obligate the U.S. Government. The fact that the Government formulated or supplied the drawings, specifications, or other data does not license the holder or any other person or corporation; or convey any rights or permission to manufacture, use, or sell any patented invention that may relate to them.

This report was cleared for public release by the Air Force Research Laboratory [insert TD site] Public Affairs Office and is available to the general public, including foreign nationals. Copies may be obtained from the Defense Technical Information Center (DTIC) (http://www.dtic.mil).

\section{AFRL-RD-PS-TR-2014-0036 HAS BEEN REVIEWED AND IS APPROVED FOR PUBLICATION IN ACCORDANCE WITH ASSIGNED DISTRIBUTION STATEMENT.}

//Signed//

DAN K. MARKER, DR-III

Project Officer $\angle /$ Signed $/ /$

KENTON T. WOOD, DR-IV, DAF

Chief, Laser Division

This report is published in the interest of scientific and technical information exchange, and its publication does not constitute the Government's approval or disapproval of its ideas or findings.

*Disseminated copies will show "//signature//" stamped or typed above the signature blocks. 


\section{REPORT DOCUMENTATION PAGE}

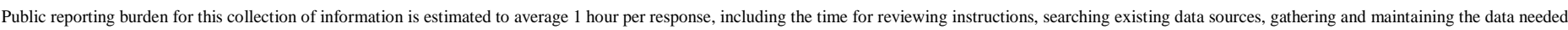

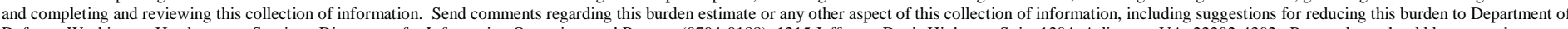

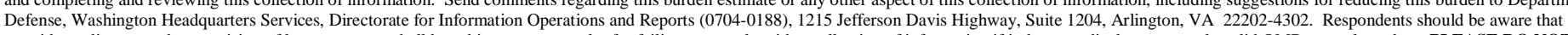

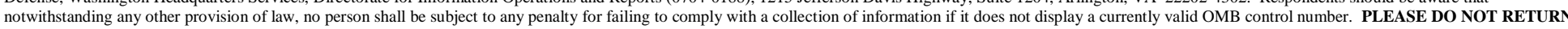
YOUR FORM TO THE ABOVE ADDRESS.

\section{REPORT DATE (DD-MM-YYYY) \\ 31-07-2014 \\ 4. TITLE AND SUBTITLE \\ ADVANCED CONTROL FILTERING AND PREDICTION FOR PHASED ARRAYS IN DIRECTED ENERGY SYSTEMS \\ 2. REPORT TYPE \\ Final Report}

(1)

\section{AUTHOR(S)}

James Steve Gibson

\section{5b. GRANT NUMBER}

5c. PROGRAM ELEMENT NUMBER

$62605 \mathrm{~F}$

5d. PROJECT NUMBER

\section{PERFORMING ORGANIZATION NAME(S) AND ADDRESS(ES)} AND ADDRESS(ES)

University of California, Los Angeles

Office of Research Administration

1100 Kinross Avenue, Ste 102

Los Angeles, CA 90095-2000

9. SPONSORING / MONITORING AGENCY NAME(S) AND ADDRESS(ES)

Air Force Research Laboratory

3550 Aberdeen Ave SE

Kirtland AFB, NM 87117-5776

\section{5f. WORK UNIT NUMBER}

D071

8. PERFORMING ORGANIZATION REPORT NUMBER

12. DISTRIBUTION / AVAILABILITY STATEMENT

Approved for public release; distribution unlimited. 377ABW-2014-1027; Jan 8, 2015.

\section{SUPPLEMENTARY NOTES}

\section{ABSTRACT}

UCLA research under this project has developed advanced methods for control, filtering, prediction and system identification in adaptive optics, laser beam pointing and target tracking. These methods can achieve significant improvements in on-target Strehl ratios and tracking jitter for phased-array high energy laser systems.

\section{SUBJECT TERMS}

Optics, Strehl, jitter, phased-array, high energy laser

\begin{tabular}{|c|c|c|c|c|c|}
\hline \multicolumn{3}{|c|}{ 16. SECURITY CLASSIFICATION OF: } & \multirow{2}{*}{$\begin{array}{l}\text { 17. LIMITATION } \\
\text { OF ABSTRACT } \\
\text { SAR }\end{array}$} & \multirow{2}{*}{$\begin{array}{l}\text { 18. NUMBER } \\
\text { OF PAGES } \\
\qquad 17\end{array}$} & \multirow{2}{*}{$\begin{array}{l}\text { 19a. NAME OF RESPONSIBLE PERSON } \\
\text { Dan K. Marker } \\
\text { 19b. TELEPHONE NUMBER (include area } \\
\text { code) }\end{array}$} \\
\hline $\begin{array}{l}\text { a. REPORT } \\
\text { Unclassified }\end{array}$ & $\begin{array}{l}\text { b. ABSTRACT } \\
\text { Unclassified }\end{array}$ & $\begin{array}{l}\text { c. THIS PAGE } \\
\text { Unclassified }\end{array}$ & & & \\
\hline
\end{tabular}




\section{TABLE OF CONTENTS}

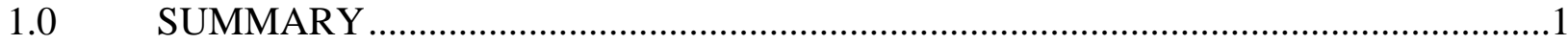

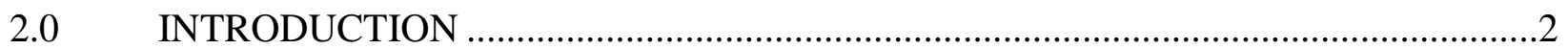

3.0 METHODS, ASSUMPTIONS, AND PROCEDURES ………………..........................

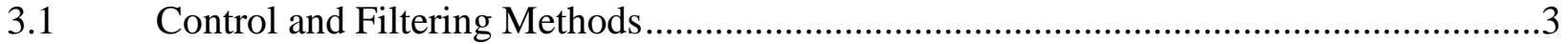

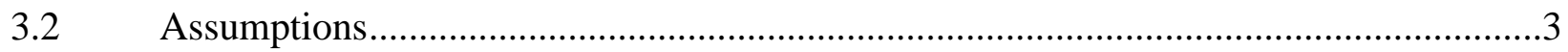

3.3 Design, Analysis and Simulation Procedures ................................................................

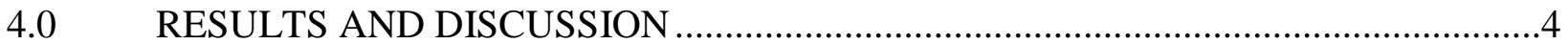

4.1 Feedback Control of Phase Ramp with Kalman Prediction from Wrapped Phase

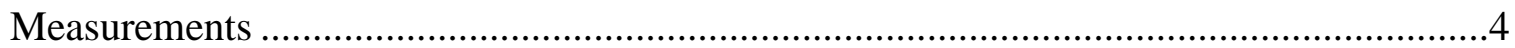

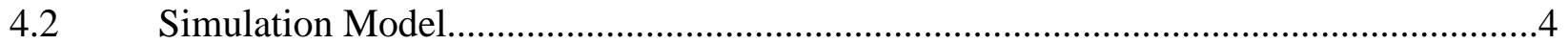

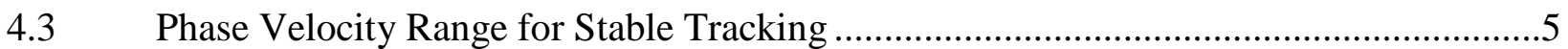

4.4 Effect of Sensor Noise and Velocity Variation on Closed-loop Error and Velocity

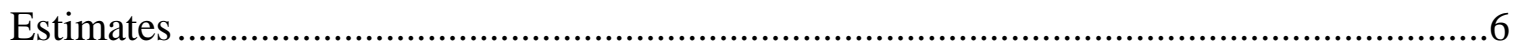

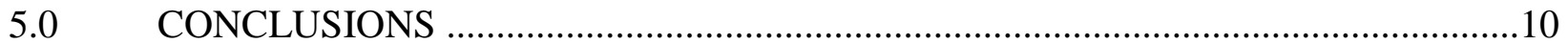

Approved for public release; distribution unlimited. 


\section{LIST OF FIGURES}

Figure 1: SIMULINK model for prediction and feedback control of a phase ramp. Mirror represented by integrator with sample time tsim. The model shown has a measurement delay equal to the length of two control sample intervals.

Figure 2: Closed-loop phase error $/ 2 \pi$. Measurement delay $=2$ control sample intervals.

Velocity vr $=0.75 \times 2 \pi /$ control sample interval. Blue: constant velocity, no sensor noise. Red: velocity standard deviation $=0.1 \times 2 \pi$, sensor noise standard deviation $=$ $0.1 \times 2 \pi$. Phase error sampled at the fast simulation rate. .........................................6

Figure 3: Closed-loop velocity estimate $/ 2 \pi$. Measurement delay $=2$ control sample intervals. Velocity $\mathrm{vr}=0.75 \times 2 \pi /$ control sample interval. Blue: constant velocity, no sensor noise. Red: velocity standard deviation $=0.1 \times 2 \pi$, sensor noise standard deviation $=$ $0.1 \times 2 \pi$. Phase error sampled at the fast simulation rate

Figure 4: Closed-loop phase error / $2 \pi$. Measurement delay $=2$ control sample intervals. Velocity $\mathrm{vr}=0.75 \times 2 \pi /$ control sample interval. Blue: constant velocity, no sensor noise. Red: velocity standard deviation $=0.1 \times 2 \pi$, no sensor noise. Phase error sampled at the fast simulation rate

Figure 5: Closed-loop velocity estimate / $2 \pi$. Measurement delay -2 control sample intervals. Velocity $\mathrm{vr}=0.75 \times 2 \pi /$ control sample interval. Blue: constant velocity, no sensor noise. Red: velocity standard deviation $=0.1 \times 2 \pi$, no sensor noise. Phase error sampled at the fast simulation rate 


\subsection{SUMMARY}

University of California, Los Angeles (UCLA) research under this project has developed advanced methods for control, filtering, prediction and system identification in adaptive optics, laser beam pointing and target tracking. These methods can achieve significant improvements in on-target Strehl ratios and tracking jitter for phased-array high energy laser systems. The main technical contribution of the research under this grant is a method for phase unwrapping, prediction and correction in phased arrays. The new method combines phase unwrapping logic with Kalman filtering and prediction in a feedback control loop. Analysis and simulation have shown that the new method can track and correct optical phase with slowly varying phase velocity and that the method is reasonably robust to sensor noise. Following is a detailed discussion of this method and representative simulation results.

Approved for public release; distribution unlimited. 


\subsection{INTRODUCTION}

Under this grant, research at UCLA has developed advanced methods for control, filtering, prediction and system identification in adaptive optics, laser beam pointing and target tracking. These methods can achieve significant improvements in on-target Strehl ratios and tracking jitter for phased-array high energy laser systems.

A primary objective of the proposed research is to develop novel methods for wavefront prediction and correction and reduction of tilt jitter, such as two methods that increase bandwidths of wavefront correction and jitter control. An equally important objective will be to develop analysis and simulation tools to evaluate closed-loop performance of phased array systems with a variety of control system designs and subject to realistic disturbances, measurement and reconstruction errors and system latencies.

The main technical contribution of the research under this grant is a method for phase unwrapping, prediction and correction in phased arrays. The new method combines phase unwrapping logic with Kalman filtering and prediction in a feedback control loop. Analysis and simulation have shown that the new method can track and correct optical phase with slowly varying phase velocity and that the method is reasonably robust to sensor noise. Following is a detailed discussion of this method and representative simulation results.

Approved for public release; distribution unlimited 


\subsection{METHODS, ASSUMPTIONS, AND PROCEDURES}

\subsection{Control and Filtering Methods}

The primary methods used to design the predictor and feedback control loop are the following:

- Kalman filtering and prediction

- Feedback control

- $\quad$ Phase Unwrapping

\subsection{Assumptions}

It is assumed that a possibly noisy measurement of wrapped optical phase is available to the digital feedback control system.

\subsection{Design, Analysis and Simulation Procedures}

The research procedure consists of designing the predictor, phase unwrapping logic and feedback control law, analyzing the closed-loop system theoretically to guarantee stability and simulating the closed-loop system in SIMULINK.

Approved for public release; distribution unlimited 


\subsection{RESULTS AND DISCUSSION}

\subsection{Feedback Control of Phase Ramp with Kalman Prediction from Wrapped Phase Measurements}

This discussion summarizes results from a study of mod $4 \pi$ phase measurement and the effects of sensor noise and velocity variation on the estimation, prediction and control of a phase ramp. Figure 1 shows a closed-loop system with two rates, one rate for simulation and the second rate for the controller. The mirror is represented by an integrator, so that the control command is a rate command to the mirror. This model assumes that the position of the mirror can be measured and used by the control loop, although this measurement probably is not necessary.

\subsection{Simulation Model}

There are three differences between the current SIMULINK model and the previous models: The measurement is mod $4 \pi$ in the current model instead of $\bmod 2 \pi$ in the previous models, white sensor noise is added to the phase measurement before the $4 \pi$ block, and the current model provides for a band-limited disturbance to be added to the phase velocity. The results discussed here were generated with only the white sensor noise. The study with velocity disturbance is in progress and will be reported later.

The controller consists of the blue blocks in Figure 1. The open-loop phase is the signal to be predicted and corrected. The Kalman predictor and unwrapping function take the delayed closed-loop phase error (mod $4 \pi$ ) as the only input, and generate a prediction of the open-loop phase. This prediction is subtracted from the true open-loop phase to form the closed-loop phase error.

The sample-and-hold rate for control is defined to be tcontr $=1$. The simulation rate, denoted by tsim, normally is some fraction of tcontr. For the simulation results in the subsequent figures, $t \operatorname{sim}=1 / 40$, and the measurement delay is either one control sample interval (= 40 tsim) or two control samples (= 40 tsim). A few simulations with $t \operatorname{sim}=1 / 200$ yielded essentially identical results to those presented here, so $t \operatorname{sim}=1 / 40$ was used for most of the numerous simulations of which the results here are representative.

Approved for public release; distribution unlimited 


\subsection{Phase Velocity Range for Stable Tracking}

The most important parameter is the ratio of the phase velocity $v r$ to the control sample interval. Since that sample interval is 1 in this model, the important ratio is $v r$. For the mod $4 \pi$ phase measurement, the current controller can track the phase and produce a reliably stable closed-loop error for phase velocities with magnitude up to $0.75 \times 2 \pi$ / control sample period. The closed-loop track loop is stable for somewhat larger velocities for sufficiently small sensor noise and some values of the initial phase. This suggests that further refinement of the Kalman predictor and control loop might produce a stable track loop for velocities somewhat larger than $0.75 \times 2 \pi$, but possibly not.

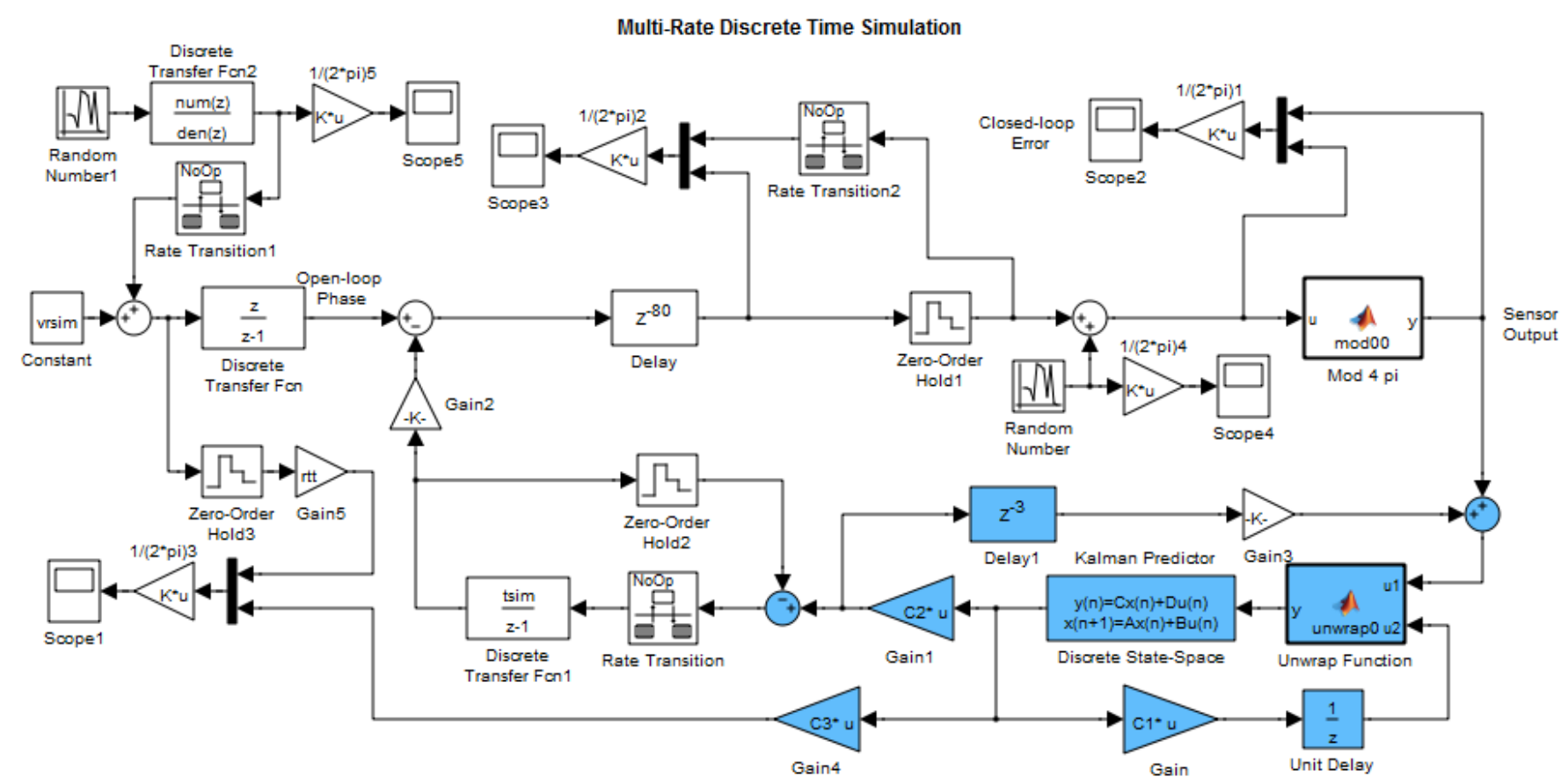

Figure 1. SIMULINK model for prediction and feedback control of a phase ramp. Mirror represented by integrator with sample time tsim. The model shown has a measurement delay equal to the length of two control sample intervals.

Approved for public release; distribution unlimited 


\subsection{Effect of Sensor Noise and Velocity Variation on Closed-loop Error and Velocity}

\section{Estimates}

Figures 2 and 3 show, respectively, the closed-loop phase error and velocity estimate for $v r=0.75 \times 2 \pi /$ control sample period (approximately the maximum velocity that this controller can track with reliable stability) and zero-mean white sensor noise. The measurement delay was two control sample periods and the sensor noise and velocity both had standard deviation = $0.1 \times 2 \pi$. (The standard deviation of the sensor noise equals the RMS value, since the noise has zero mean.) For Figures 4 and 5, the velocity had standard deviation $=0.1 \times 2 \pi$ but there was no sensor noise.

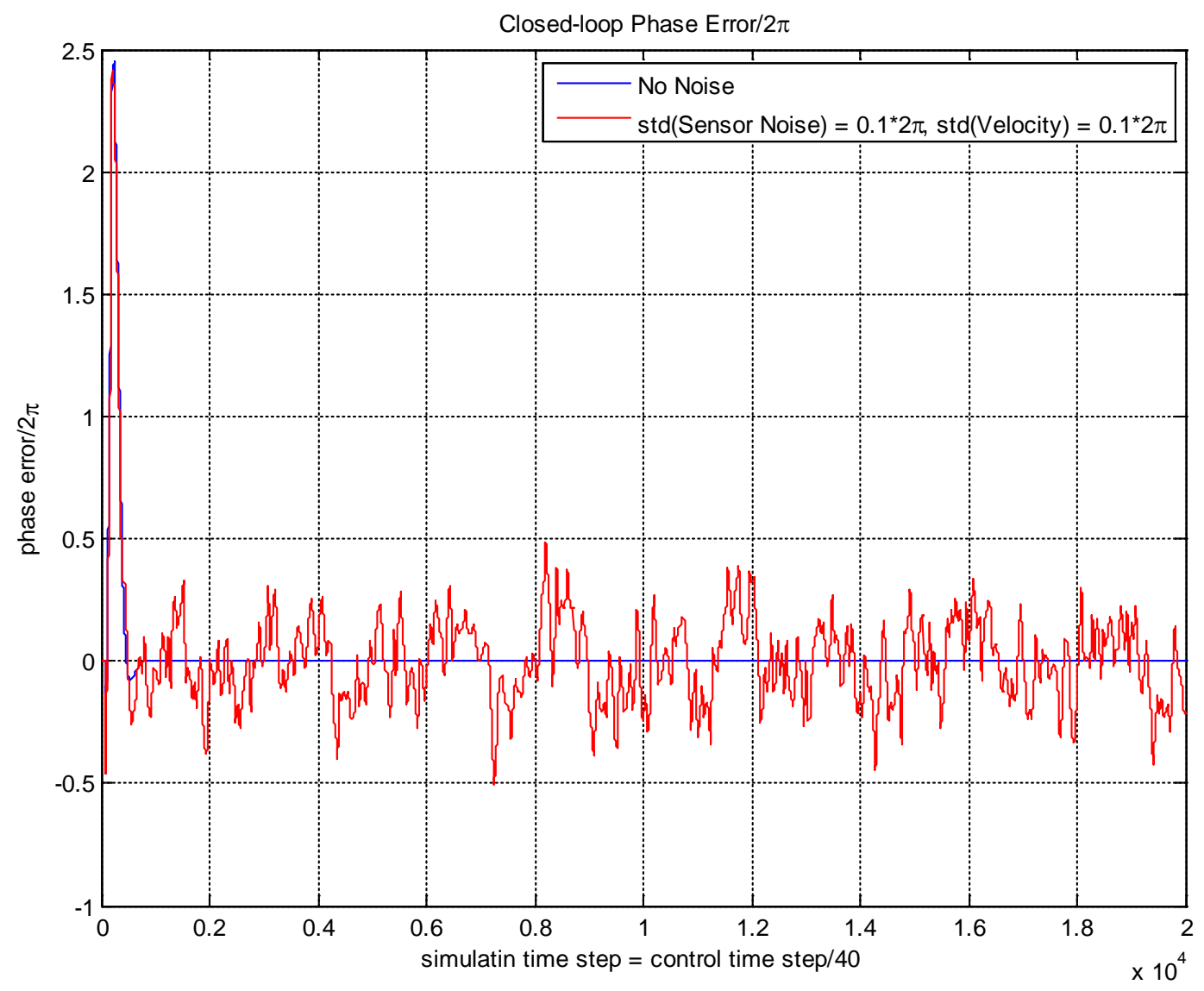

Figure 2. Closed-loop phase error $/ 2 \pi$. Measurement delay $=2$ control sample intervals. Velocity $v r=0.75 \times 2 \pi$ / control sample interval. Blue: constant velocity, no sensor noise. Red: velocity standard deviation $=0.1 \times 2 \pi$, sensor noise standard deviation $=0.1 \times 2 \pi$. Phase error sampled at the fast simulation rate.

Approved for public release; distribution unlimited 


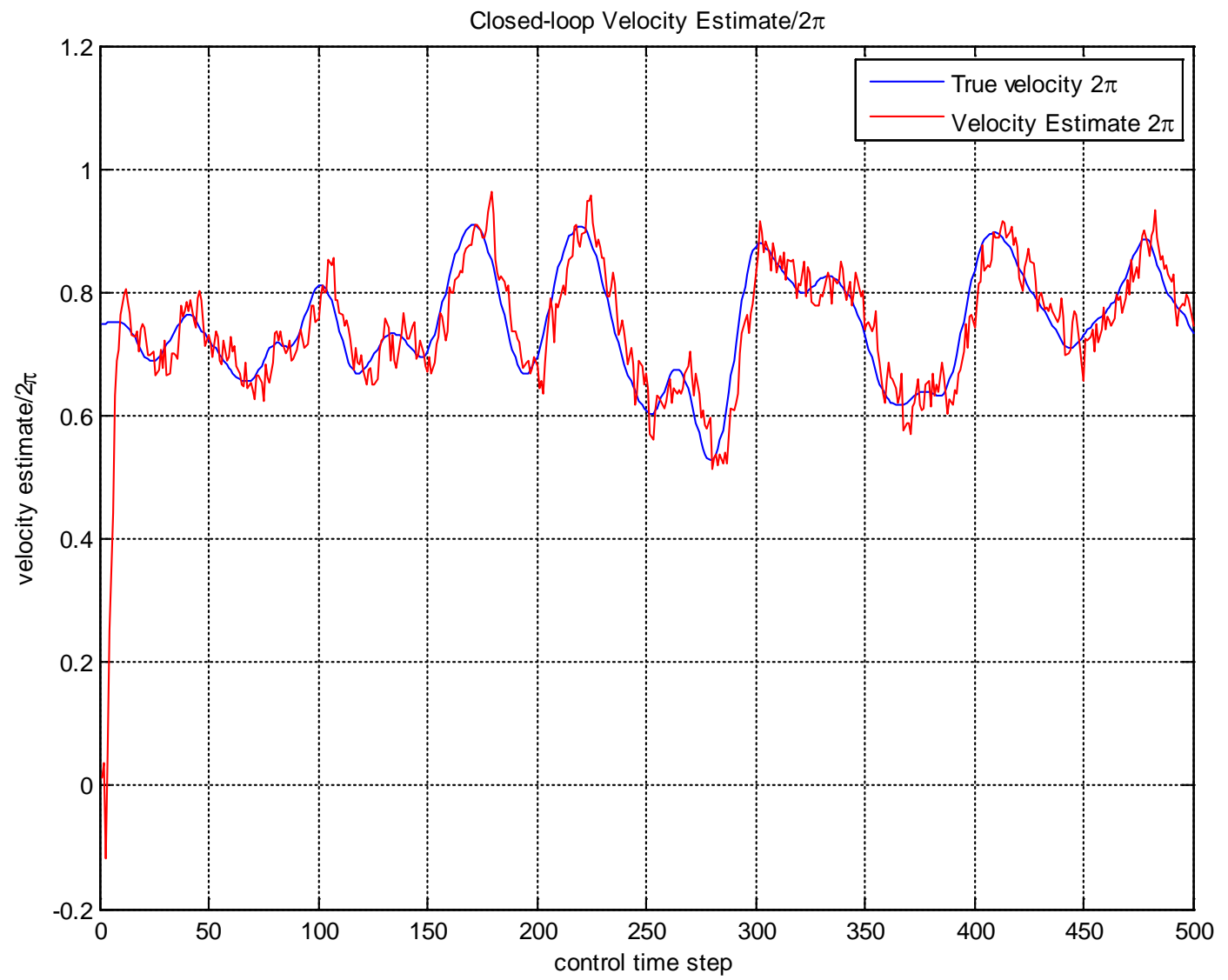

Figure 3. Closed-loop velocity estimate $/ 2 \pi$. Measurement delay $=2$ control sample intervals. Velocity $v r=0.75 \times 2 \pi$ / control sample interval. Blue: constant velocity, no sensor noise. Red: velocity standard deviation $=0.1 \times 2 \pi$, sensor noise standard deviation $=0.1 \times 2 \pi$. Phase error sampled at the fast simulation rate.

Approved for public release; distribution unlimited 


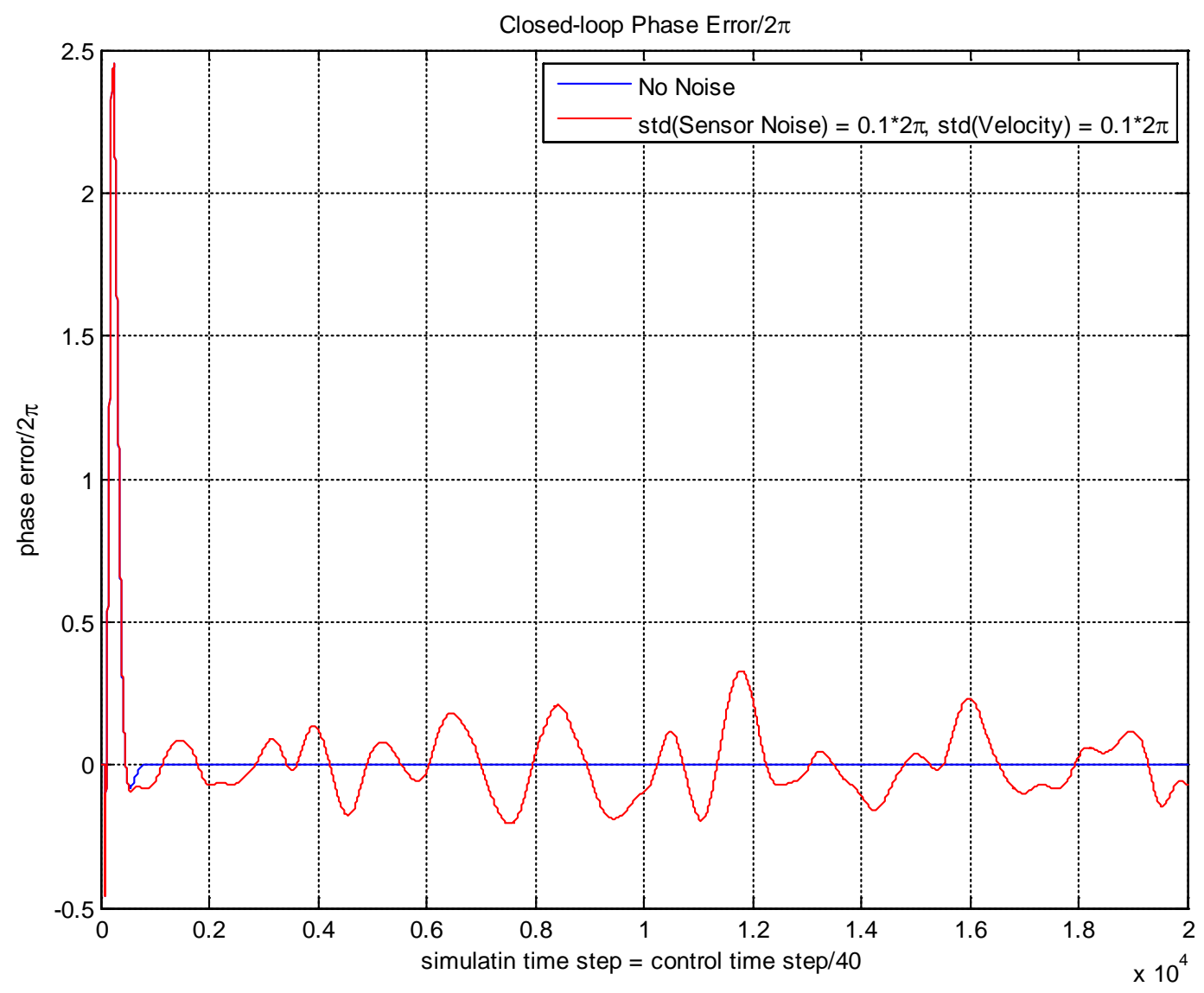

Figure 4. Closed-loop phase error $/ 2 \pi$. Measurement delay $=2$ control sample intervals. Velocity $v r=0.75 \times 2 \pi$ / control sample interval. Blue: constant velocity, no sensor noise. Red: velocity standard deviation $=0.1 \times 2 \pi$, no sensor noise. Phase error sampled at the fast simulation rate.

Approved for public release; distribution unlimited 


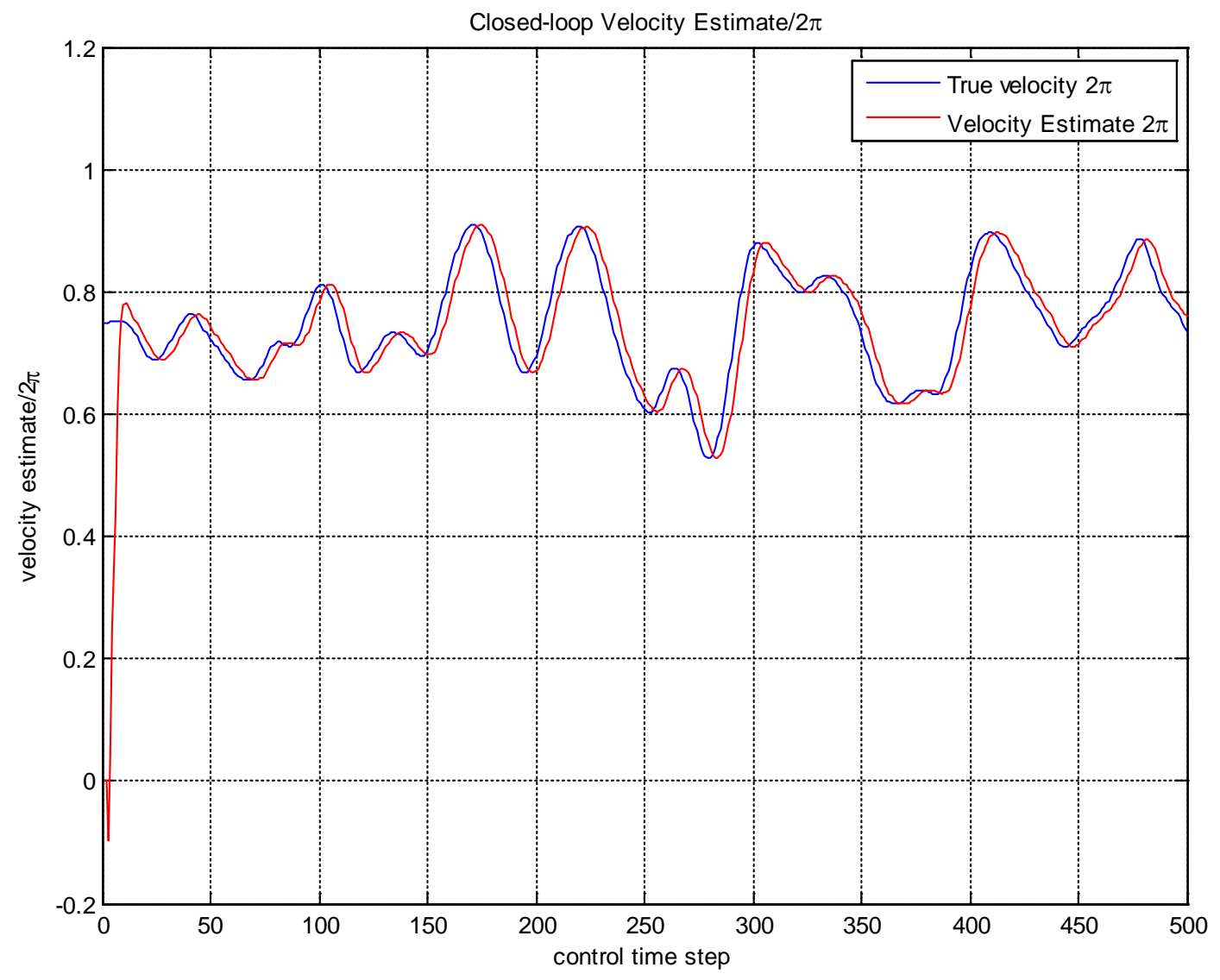

Figure 5. Closed-loop velocity estimate $/ 2 \pi$. Measurement delay $=2$ control sample intervals. Velocity $v r=0.75 \times 2 \pi$ / control sample interval. Blue: constant velocity, no sensor noise. Red: velocity standard deviation $=0.1 \times 2 \pi$, no sensor noise. Phase error sampled at the fast simulation rate. 


\subsection{CONCLUSIONS}

This project has developed advanced methods for control, filtering, prediction and system identification in adaptive optics, laser beam pointing and target tracking. These methods can achieve significant improvements in on-target Strehl ratios and tracking jitter for phased-array high energy laser systems. The main technical contribution of the research under this grant is a method for phase unwrapping, prediction and correction in phased arrays. The new method combines phase unwrapping logic with Kalman filtering and prediction in a feedback control loop. Analysis and simulation have shown that the new method can track and correct optical phase with slowly varying phase velocity and that the method is reasonably robust to sensor noise. Further research should extend the methods developed here to multiple apertures and higher-order wavefront correction in phased-array systems.

Approved for public release; distribution unlimited 


\section{DISTRIBUTION LIST}

DTIC/OCP

8725 John J. Kingman Rd. Suite 0944

Ft. Belvoir, VA 22060-6218

1 cy

AFRL/RVIL

Kirtland AFB, NM 87117-5776

$1 \mathrm{cy}$

Dan Marker

Official Record Copy

AFRL/RDLTS

1 cy

Approved for public release; distribution unlimited 